\title{
Controllable chemical modification of Polyaniline Nanofibres
}

\author{
Emer Lahiff $^{1^{*}}$, Carol Lynam ${ }^{2}$, Niamh Gilmartin ${ }^{2}$, Gordon Wallace ${ }^{3}$, Richard O'Kennedy ${ }^{2}$ and \\ Dermot Diamond ${ }^{1}$ \\ ${ }^{1}$ CLARITY: The Centre for Sensor Web Technologies, Dublin City University, Dublin 9, \\ Ireland. \\ ${ }^{2}$ School of Biotechnology and Biomedical Diagnostics Institute, National Centre for Sensor \\ Research, Dublin City University, Dublin 9, Ireland. \\ ${ }^{3}$ ARC Centre of Excellence for Electromaterials Science, Intelligent Polymer Research \\ Institute, University of Wollongong, Wollongong, NSW 2522, Australia.
}

\begin{abstract}
A method for simply and controllably modifying the surface of polyaniline nanofibres is described. The technique can be used to attach substituents bearing both acid and amine functional groups, making the materials suitable for further modification. Acid/amine functionalisation is achieved by a simple reflux reaction and therefore is a quick and easily scalable process. The modified nanofibres maintain their ability to switch between different states displaying distinctly different properties, thus making them suitable for adaptive sensing applications. As an example, we demonstrate how biomolecules can be attached to these functionalised nanofibres, to produce conducting polymer-based biosensors.
\end{abstract}

\section{INTRODUCTION}

Polyaniline (PAni) is an example of a stable conducting polymer whose properties (optical, electrical and electrochemical) change in response to the immediate molecular environment of the material. PAni can be cheaply and conveniently synthesized and, thus, has huge potential in sensor applications. PAni nanofibres can be synthesised directly by chemical means. The technique used here is known as interfacial polymerisation and it is a scalable technique whereby increasing the amount of reactants results in a greater yield of nanofibre product. By using PAni nanofibres (versus bulk) the surface area exposed to the target molecules can be dramatically increased, resulting in a sensor material with enhanced sensitivity and improved response times ${ }^{1-5}$. Our focus is on the covalent modification of these structures post-polymerisation, while simultaneously maintaining the intrinsic nanomorphology of the polymer material ${ }^{6}$. Using the technique described, control over the extent of functionalisation can be achieved ${ }^{7}$. Functionalised PAni nanofibres can then be used as templates for further modification with target specific receptor sites, thus improving selectivity. Here we demonstrate the subsequent attachment of antibody based bioreceptors, whereby resulting PAni-antibody conjugates have applications in the field of electrochemical immunosensors.

\section{EXPERIMENT}




\section{Nanofibre Synthesis}

Polyaniline nanofibres were synthesised by interfacial polymerisation between an aqueous and an organic layer, as reported previously ${ }^{1}$. The product was purified by centrifugation ( $4000 \mathrm{rpm} / 5 \mathrm{~min} / 3$ cycles) and suspended as a colloid in deionised water. Fibres were then modified with either acid- or amine-terminated thiols $\left(\mathrm{HS}\left(\mathrm{CH}_{2}\right)_{3} \mathrm{COOH}\right.$ or $\left.\mathrm{HS}\left(\mathrm{CH}_{2}\right)_{2} \mathrm{NH}_{2}\right)$, by refluxing at $100{ }^{\circ} \mathrm{C}$ for 2 hours in an aqueous buffer at $\mathrm{pH} 4^{8}$. All experiments were carried out under identical conditions using $45 \mathrm{mg}$ PAni. The product was purified by centrifugation ( $4000 \mathrm{rpm} / 5 \mathrm{~min} / 3$ cycles) and re-suspended as a colloid in deionised water.

\section{Antibody immobilisation}

Antibodies were subsequently covalently immobilised by the addition of mouse $\operatorname{IgG}(100 \mu \mathrm{l}$, $0.1 \mathrm{mg} / \mathrm{ml}$ ) and ethyl diaminocarbodiimide $(4 \mathrm{mg})$, to a dispersion of PAni nanofibres $(1 \mathrm{ml}$, $0.2 \mathrm{mg} / \mathrm{ml})$. The dispersion was left shaking $(200 \mathrm{rpm})$ at $37^{\circ} \mathrm{C}$ for 1 hour. The PAni-IgG conjugate was centrifuged and washed in distilled water 3 times. Any remaining un-reacted sites on the nanofibres were blocked with $5 \mathrm{wt}$. $\%$ milk marvel overnight at $4{ }^{\circ} \mathrm{C}$. The PAni$\mathrm{IgG}$ conjugate was then centrifuged $(10 \mathrm{~min}$ at $4000 \mathrm{rpm})$ and washed 3 times with distilled water. Anti-mouse IgG labeled with horse radish peroxidase (HRP) was diluted 1 in 1000 in 1 $\%(\mathrm{w} / \mathrm{v})$ milk marvel/PBS $(0.1 \mathrm{M}, \mathrm{pH} 7.4)$ to give a final concentration of $0.6 \mu \mathrm{g} / \mathrm{ml}$ and $1 \mathrm{ml}$ of this solution was added to the PAni-IgG pellet, re-dispersed and incubated at $37^{\circ} \mathrm{C}$ for one hour. The PAni mixture was then centrifuged (10 $\mathrm{min}$ at $4000 \mathrm{rpm})$ and washed a further 3 times with water and the PAni pellet re-suspended in $0.5 \mathrm{ml}$ water.

\section{Characterisation}

Modified nanofibres were coated with $10 \mathrm{~nm} \mathrm{Au} / \mathrm{Pd}$ and imaged using field emission scanning electron microscopy (FESEM) at an accelerating voltage of $20 \mathrm{kV}$ on a S-4300 Hitachi system. UV-vis spectroscopy was used to monitor the switching of modified PAni between different redox states. It was carried out on a Perkin Elmer UV-vis NIR Lambda 900 Spectrometer at $1 \mathrm{~nm}$ resolution. Cyclic voltammetry was used to confirm HRP labelled secondary-antibody attachment to the modified Pani nanofibres. It was performed using an eDAQ e-corder (401) and potentiostat/galvanostat (EA 160) with Chart v5.1.2/EChem v 2.0.2 software (ADInstruments), and a PC computer, in phosphate buffered saline solution (PBS $0.1 \mathrm{M}, \mathrm{pH}$ 7.4) with $\mathrm{Ag} / \mathrm{AgCl}$ reference electrode and platinum mesh counter electrode.

\section{DISCUSSION}

Interfacial polymerisation resulted in the formation of polyaniline nanofibres. These nanofibres could be purified by a simple centrifugation technique and subsequently suspended in a solvent of choice. Nanofibres could be modified by a simple reflux process to attach thiol terminated carboxylic acid or amine side-chains. The covalent attachment was verified using FTIR and NMR as previously published by the authors ${ }^{7}$. Post-modification, nanofibres maintain their nanostructure (Figure 1), and hence high surface area. They can be cast as films onto a variety of substrates, or used in solution. 

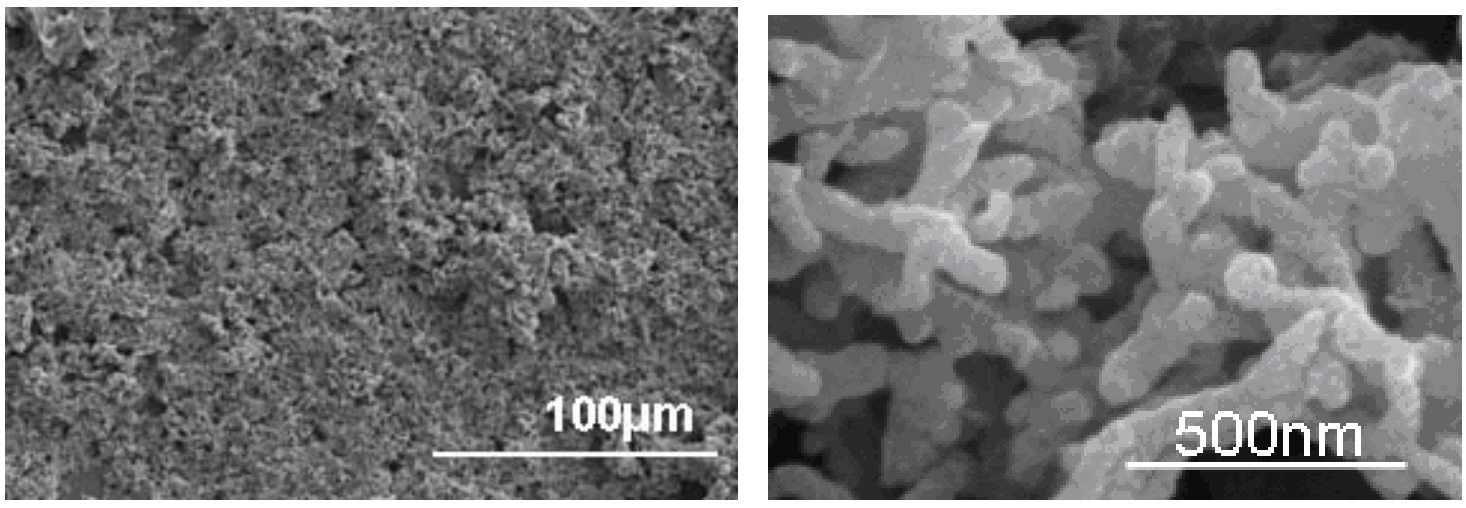

Figure 1: SEM imaging confirms that PAni is nanostructured post-functionalisation. The image on the left is magnified $\times 500$ and the one on the right is $\mathrm{x} 100$.

Polyaniline nanofibres are of interest for sensing applications as they can adapt and respond in an intelligent manner to their immediate surroundings. As they respond, the chemical structure of the material is altered and this is reflected as a change in the optical properties such as colour. Post-functionalisation, nanofibres maintain this ability to switch states hence making them suitable for sensing applications. Like PAni, the colour of the functionalised fibres is sensitive to the environment of the material (Figure 2). This colour change can be detected using UV-vis spectroscopy.
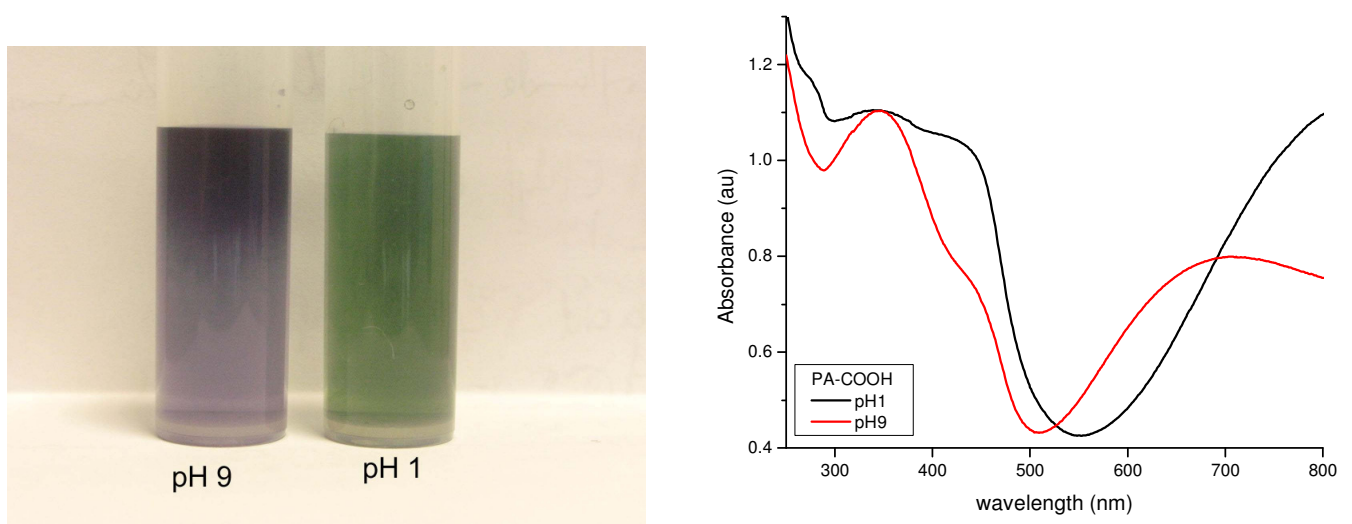

Figure 2: The optical properties of carboxylic acid modified nanofibres (PAni-COOH) change in response to the $\mathrm{pH}$ of their immediate chemical environment. The colour of a PAni-COOH dispersion changes from purple to green as the $\mathrm{pH}$ decreases (this is the same as the colour change observed for unmodified PAni). The UV-vis spectrum of the material also changes accordingly.

\section{Covalent attachment of biomolecules}

One of the major limitations for commercial application of PAni sensors is a lack of selectivity. By functionalizing PAni, we create a platform that can be further modified to attach more sophisticated molecules, such as biomolecules ${ }^{9}$. Thus providing a simple method for improving selectivity of the material. Both amine and acid side-chains can be used as 
anchor sites to attach other molecules. Here we show how antibodies can be covalently attached to these functionalised nanofibres. Initial studies were performed using mouse IgG. Post-modification the nanofibre surface morphology changes due to the covalent antibody attachment (Figure 3). However, the material maintains a high surface area (as seen in SEM), which is significant for sensing applications as it enables a greater number of reaction sites to be accessed. The electrochemical surface area of the material may be calculated from the charge density obtained from cyclic voltammetry. No significant decrease in the electrochemical surface area is observed for nanofibres pre and post-modification with biomolecules (not shown). Covalently bound antibodies could be used to detect the presence of secondary antibodies (a-IgG). This was demonstrated electrochemically by using HRPlabeled secondary antibodies. The iron redox couple (A and A') of the HRP can be clearly seen in cyclic voltammetry scans at $0.2 \mathrm{~V}$ (this is absent for the PAni nanofibres without antibodies attached).
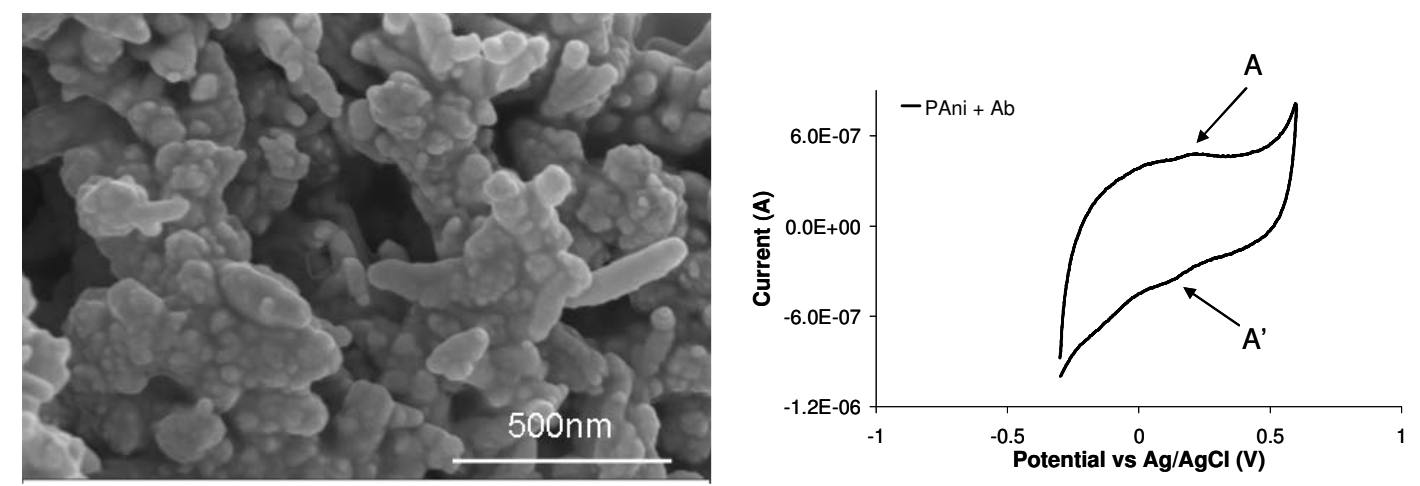

Figure 3: FESEM (left) shows that the nanostructure is maintained after antibody attachment. An iron redox couple ( $\mathrm{A}$ and $\mathrm{A}^{\prime}$ ) centered at $0.2 \mathrm{~V}$ confirms the presence of secondary antibodies. This peak is absent for the PAni control experiment.

\section{CONCLUSIONS}

This study shows the successful functionalization of PAni nanofibres. By focusing on the modification of nanofibre surfaces post-polymerisation, the intrinsic properties (such as doping) of the PAni backbone can be maintained. Modified PAni nanofibres provide a scaffold which can be further modified with selective moieties for the detection of target species, such as biomolecules. Using the process described, we can combine the advantages of a functionalized organic conductor and a low dimensional nanostructure.

\section{ACKNOWLEDGMENTS}

The authors acknowledge funding from Science Foundation Ireland under SFI 07/CE/I1 147 "CLARITY: Centre for Sensor Web Technologies", and also Enterprise Ireland under PC/2008/0149. CL acknowledges the EU Seventh Framework Programme for support in the 
form of a Marie Curie Re-Integration Grant. This material is based upon research supported by the Science Foundation Ireland under Grant No. 05/CE3/B754.

\section{REFERENCES}

1. Huang, J. X.; Virji, S.; Weiller, B. H.; Kaner, R. B., Polyaniline nanofibers: Facile synthesis and chemical sensors. Journal of the American Chemical Society 2003, 125, (2), 314-315.

2. $\quad$ Virji, S.; Kaner, R. B.; Weiller, B. H., Hydrogen sensors based on conductivity changes in polyaniline nanofibers. Journal of Physical Chemistry B 2006, 110, (44), 2226622270.

3. Virji, S.; Kaner, R. B.; Weiller, B. H., Hydrazine detection by polyaniline using fluorinated alcohol additives. Chemistry of Materials 2005, 17, (5), 1256-1260.

4. Virji, S.; Huang, J. X.; Kaner, R. B.; Weiller, B. H., Polyaniline nanofiber gas sensors: Examination of response mechanisms. Nano Letters 2004, 4, (3), 491-496.

5. Virji, S.; Fowler, J. D.; Baker, C. O.; Huang, J. X.; Kaner, R. B.; Weiller, B. H., Polyaniline manofiber composites with metal salts: Chemical sensors for hydrogen sulfide. Small 2005, 1, (6), 624-627.

6. Lahiff, E., Bell, S, Diamond, D., Functionalised Nanostructured Polyaniline - A New Substrate for Building Adaptive Sensing Surfaces. Mater. Res. Soc. Symp. Proc. 2008, 1054, 1054-FF05-05.

7. Lahiff, E.; Woods, T.; Blau, W.; Wallace, G. G.; Diamond, D., Synthesis and characterisation of controllably functionalised polyaniline nanofibres. Synthetic Metals 2009, 159, (7-8), 741-748.

8. Lahiff, E., Scarmagnani, S, Schazmann, B, Cafolla, A, Diamond, D., Covalent Attachment of Functional Side-Groups to Polyaniline Nanofibres. International Journal of Nanomanufacturing. 2010, 5, (1/2), 88-99.

9. Lahiff, E., Lynam, C, Gilmartin, N, Diamond, D, O'Kennedy, R., Polyaniline nanofibre transducers for Immunoassays. Nanoscale 2009, Submitted. 